\title{
SOIL MOISTURE RETRIEVAL UNDER AGRICULTURAL VEGETATION USING FULLY POLARIMETRIC SAR
}

\author{
Thomas Jagdhuber $^{1,2}$, Irena Hajnsek ${ }^{1,3}$, Konstantinos P. Papathanassiou ${ }^{1}$, Axel Bronstert ${ }^{2}$ \\ ${ }^{1}$ German Aerospace Center, Microwaves and Radar Institute, PO Box 1116, 82234 Wessling \\ ${ }^{2}$ University of Potsdam, Institute of Earth and Environmental Sciences, Karl-Liebknecht-Strasse 24-25, 14476 Potsdam \\ ${ }^{3}$ ETH Zurich, Institute of Environmental Engineering, Schafmattstr. 6, 8093 Zurich \\ Email: thomas.jagdhuber@dlr.de, irena.hajnsek@dlr.de, kostas.papathanassiou@dlr.de, axelbron@uni-potsdam.de
}

\begin{abstract}
Soil moisture retrieval under agricultural vegetation is assessed by a hybrid decomposition and inversion algorithm using fully polarimetric SAR data of DLR's E-SAR system at L-band. The results for the AgriSAR and SARTEO campaigns, conducted in 2006 and 2008 within the Peene and the Rur catchment, reveal a very high inversion rate leading to a spatially continuous inversion along the entire growth cycle in agricultural areas. The validation with in situ measurements for a variety of summer and winter crops states a root mean square error of $6.25 \mathrm{vol} . \%$ and $5.77 \mathrm{vol} . \%$ respectively, while a wide moisture range $(\sim 2-30 \mathrm{vol} . \%)$ is covered by the soil moisture inversion under vegetation.
\end{abstract}

Index Terms - SAR polarimetry, L-band, soil moisture estimation, hybrid decomposition, agriculture

\section{INTRODUCTION}

The availability of soil moisture information with an appropriate spatial and temporal coverage is of particular relevance for hydrological modeling, flood forecasting and precision farming [1]. For a continuous monitoring strategy, the high-resolution, illumination- and weather-independent imaging capabilities of SAR constitute a great benefit. In particular, the sensitivity of the SAR measurements to the dielectric constant of the soil (soil moisture) enables the assessment of soil conditions on scales much larger than the field scale.

The soil moisture retrieval must consider in many cases the influence of a varying agricultural vegetation cover along the growth cycle. In this sense, the penetration capabilities of longer wavelength SAR (L-band) are combined with polarimetric decomposition techniques to develop an algorithm for soil moisture inversion under agricultural vegetation cover.

\section{METHODOLOGY OF SOIL MOISTURE RETRIEVAL UNDER VEGETATION COVER}

Soil moisture retrieval from agricultural regions along the growth season includes soil and vegetation components. Hence, three scattering components (soil surface $\left[T_{S}\right]$, dihedral $\left[T_{D}\right]$ and vegetation volume $\left[T_{V}\right]$ ) contribute to the measured coherency matrix $[T]$, as presented in Eq. 1 .

In $[2,3]$ fully polarimetric model-based decomposition algorithms are introduced for the separation of vegetation and ground scattering components for soil moisture inversion

$$
[T]=\left[T_{S}\right]+\left[T_{D}\right]+\left[T_{V}\right]
$$

Polarimetric model-based decompositions, which are the basis of this algorithm, are introduced in [4,5]. The novel hybrid decomposition, defined in Eq. 2 (see next page) and [6], is derived from a generalized form, proposed in [5], and combines a model-based with an eigen-based decomposition. In order to extract in a first step the soil components, a model-based rank-3 volume component $\left[T_{V}\right]$, representing the vegetation cover, is subtracted from the measured data $[T]$ to retrieve the two eigen-based rank-1 ground components (surface $\left[T_{S}\right]$, dihedral $\left[T_{D}\right]$ ) as described in Eq. 3 [2,6]. The model-based volume component represents a cloud of randomly oriented particles with a particle shape, described by $\alpha_{V}$ for different vegetation geometries (from spheres $\alpha_{V}=0$ to dipoles $\left.\alpha_{V}=\pi / 4\right)$

$\left[T_{S}\right]+\left[T_{D}\right]=\left[\begin{array}{ccc}T_{11} & T_{12} & 0 \\ T_{12}^{*} & T_{22} & 0 \\ 0 & 0 & T_{33}\end{array}\right]-f_{v}\left[\begin{array}{ccc}\cos \left(\alpha_{v}\right)^{2} & 0 & 0 \\ 0 & \frac{1}{2} \sin \left(\alpha_{v}\right)^{2} & 0 \\ 0 & 0 & \frac{1}{2} \sin \left(\alpha_{v}\right)^{2}\end{array}\right]$.

In this sense, the included volume intensity component $f_{v}$ leads in many cases to erroneous results and an effective vegetation model is still missing up to now [3]. In [6] a Bragg surface scattering model is incorporated in the $f_{v^{-}}$ calculus to develop a physically constraint volume intensity component $f_{v p}$.

Using the $f_{v p}$-component in a second eigen-based step, the intensity of the two different ground components $\left(f_{d}, f_{s}\right)$ are obtained with Eq. 4 representing the eigenvalues of the ground components $\left(\left[T_{S}\right]+\left[T_{D}\right]\right)$. From their respective eigenvectors, the ground scattering mechanisms $\left(\alpha_{d}, \alpha_{s}\right)$ are derived in Eq. 5. Due to an orthogonality condition of $\alpha_{d}$ 
and $\alpha_{s}$, presented in [5,6], their calculus results in a physical separation between surface and dihedral scattering, because the first alpha angle from $0^{\circ}$ to $45^{\circ}$ is allocated with surface scattering, while the second alpha angle from $45^{\circ}$ to $90^{\circ}$ is classified as dihedral scattering. In a following step $f_{d}$ and $f_{s}$ are assigned accordingly [6].

Afterwards a low parameterized electromagnetic surface scattering model (Bragg) is applied to obtain the closest match between modeled $\left(\beta_{B}\right)$ and decomposed $(\beta)$ polarimetric components for subsequent inversion [2,3].

$$
\text { Inversion with } \beta=-\tan \left(\alpha_{\mathrm{s}}\right): \quad \mathcal{E}_{s}=\min \left(\left|\beta-\beta_{\mathrm{B}}\right|\right)
$$

The Bragg model for the surface scattering mechanism $\beta_{m}$ is shown in Eq. 7 providing the link between the surface scattering mechanism $\beta_{B}$ and the horizontal and vertical Bragg reflection coefficients $\left(R_{H H}, R_{V V}\right)[2,3]$

$$
\beta_{B}=\left(R_{H H}-R_{V V}\right) /\left(R_{H H}+R_{V V}\right) .
$$

These coefficients are only a function of the incidence angle $\theta$ of the acquisition system and the dielectric constant of the soil $\varepsilon_{s}$. Finally, the dielectric constant $\mathcal{E}_{s}$ is transformed into volumetric soil moisture using the universal polynomial of Topp et al. [7].

\section{EXPERIMENTAL RESULTS}

The inversion algorithm for agricultural vegetation cover is applied on the data set of the AgriSAR and SARTEO campaigns. The AgriSAR campaign was conducted over four months in 2006 including the entire vegetation growth period [8]. The test site is located in Northern Germany within the Peene catchment. The SARTEO campaign was operated in the end of May 2008 within the Rur catchment close to Jülich, Germany [9]. Within these campaigns fully polarimetric L-band data were recorded by DLR's airborne E-SAR system with high spatial resolution (slant range: $1.5 \mathrm{~m}$, azimuth: $0.5 \mathrm{~m}$ ). Simultaneously to the data takes, soil moisture and vegetation parameters were measured on selected test fields with varying crop and soil types. Fig. 1 shows the data of a continuously recording soil moisture station, conducted by the LMU Munich for the entire growth period, together with the maximum vegetation height.

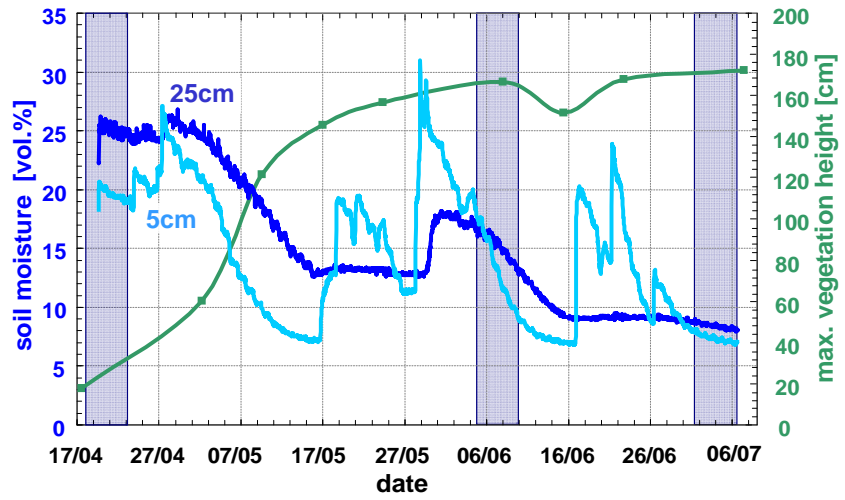

Fig. 1. Continuously recorded soil moisture in depth of $5 \mathrm{~cm}$ and $25 \mathrm{~cm}$ and maximum vegetation height for the entire growth period from April to July 2006, acquired by LMU during the AgriSAR campaign; Blue bars indicate data takes of the E-SAR system [8].

The vertical blue bars represent the dates of the E-SAR acquisitions, which track the decreasing trend in soil moisture from a level of $25 \mathrm{vol} . \%$ in April (beginning of growing season), to $15 \mathrm{vol} . \%$ in June down to 8vol.\% in July (end of growing season). In addition, the soil moisture measurements of the SARTEO campaign report a medium level of soil moisture with $21 \mathrm{vol} \%$ [9]. Hence, the retrieval algorithm is examined for a variety of soil conditions and for a distinctively growing vegetation cover (max. height: $18 \mathrm{~cm}$ (April) - 172cm (July)), which represents an optimum test bed for soil moisture inversion under agricultural vegetation cover.

As already described in [6], the hybrid decomposition with a constraint volume intensity component $f_{v p}$ allows a physically meaningful estimation of the three scattering components ([T $\left.],\left[T_{D}\right],\left[T_{V}\right]\right)$. Fig. 2 depicts the surface and dihedral scattering mechanisms $\left(\alpha_{d}, \alpha_{s}\right)$ after splitting of the ground component by the orthogonality criterion of $[5,6]$. The undisrupted and transient trend of $\alpha_{d}$ and $\alpha_{s}$ reveals the clean separation of the ground scattering mechanisms by the eigen-based orthogonality criterion, which is a prerequisite for a subsequent inversion with a continuously high inversion rate for the entire data set. The results for the soil moisture inversion under agricultural vegetation cover are displayed in Fig. 3 for the AgriSAR and the SARTEO campaign (spatial averaging on [T]: 10m x 10m).

$$
\begin{aligned}
& {[T]=f_{s}\left[\begin{array}{ccc}
\cos \left(\alpha_{s}\right)^{2} & -\cos \left(\alpha_{s}\right) \sin \left(\alpha_{s}\right) & 0 \\
-\cos \left(\alpha_{s}\right) \sin \left(\alpha_{s}\right) & \sin \left(\alpha_{s}\right)^{2} & 0 \\
0 & 0 & 0
\end{array}\right]+f_{d}\left[\begin{array}{ccc}
\sin \left(\alpha_{d}\right)^{2} & \cos \left(\alpha_{d}\right) \sin \left(\alpha_{d}\right) & 0 \\
\cos \left(\alpha_{d}\right) \sin \left(\alpha_{d}\right) & \cos \left(\alpha_{d}\right)^{2} & 0 \\
0 & 0 & 0
\end{array}\right]+\frac{f_{v}}{2}\left[\begin{array}{ccc}
2 \cos \left(\alpha_{v}\right)^{2} & 0 & 0 \\
0 & \sin \left(\alpha_{v}\right)^{2} & 0 \\
0 & 0 & \sin \left(\alpha_{v}\right)^{2}
\end{array}\right]} \\
& f_{d, s}=\frac{1}{16}\left(8 T_{11}+8 T_{22}-f_{v}\left(6+2 \cos \left(2 \alpha_{v}\right)\right) \pm \sqrt{22 f_{v}^{2}+64\left(4\left|T_{12}\right|^{2}+\left(T_{11}-T_{22}\right)^{2}\right)+32 f_{v}\left(T_{22}-T_{11}\right)+6 f_{v}\left(\left(f_{v}-4 T_{11}+4 T_{22}\right) 4 \cos \left(2 \alpha_{v}\right)+3 f_{v} \cos \left(4 \alpha_{v}\right)\right)}\right) \\
& \left.\alpha_{d, s}=\arccos \left[\left(1+256 \mid \frac{T_{12}^{*}}{2 f_{v}-8 T_{11}+8 T_{22}+6 f_{v} \cos \left(2 \alpha_{v}\right) \pm \sqrt{22 f_{v}^{2}+64\left(4\left|T_{12}\right|^{2}+\left(T_{11}-T_{22}\right)^{2}\right)+32 f_{v}\left(T_{22}-T_{11}\right)+6 f_{v}\left(\left(f_{v}-4 T_{11}+4 T_{22}\right) 4 \cos \left(2 \alpha_{v}\right)+3 f_{v} \cos \left(4 \alpha_{v}\right)\right)} \mid}\right]^{2}\right]^{-\frac{1}{2}}\right]
\end{aligned}
$$




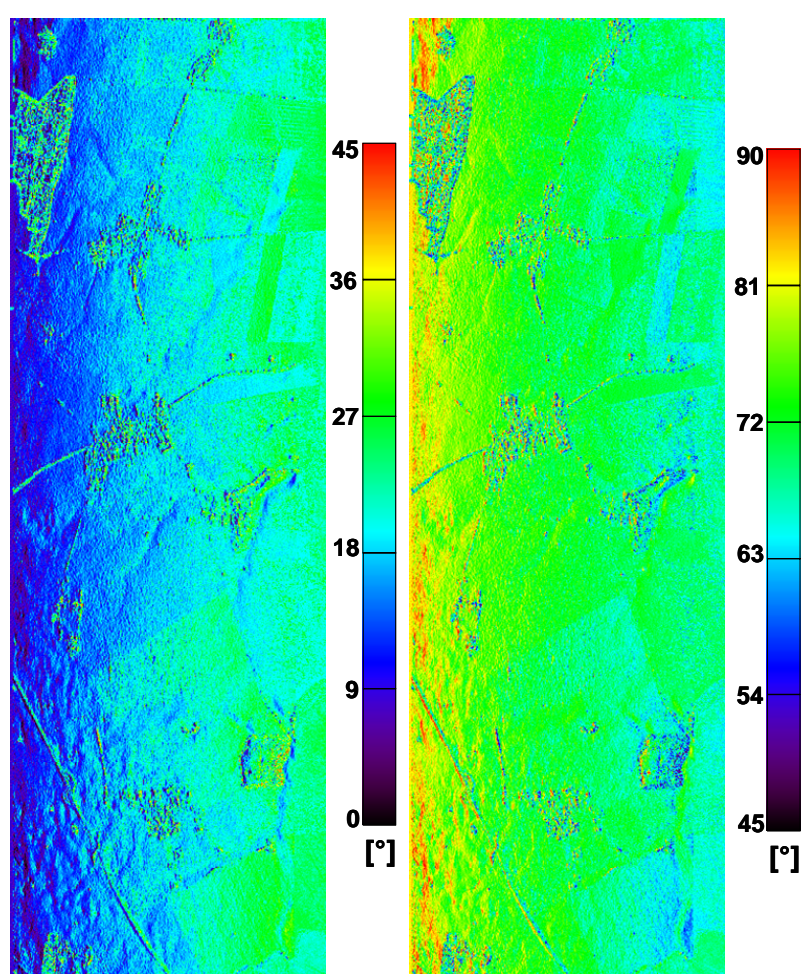

Fig. 2. Surface and dihedral scattering mechanisms $\left(\alpha_{s}, \alpha_{d}\right)$ of the April acquisition within the AgriSAR 2006 campaign, separated by the orthogonality criterion of $[5,6]$.
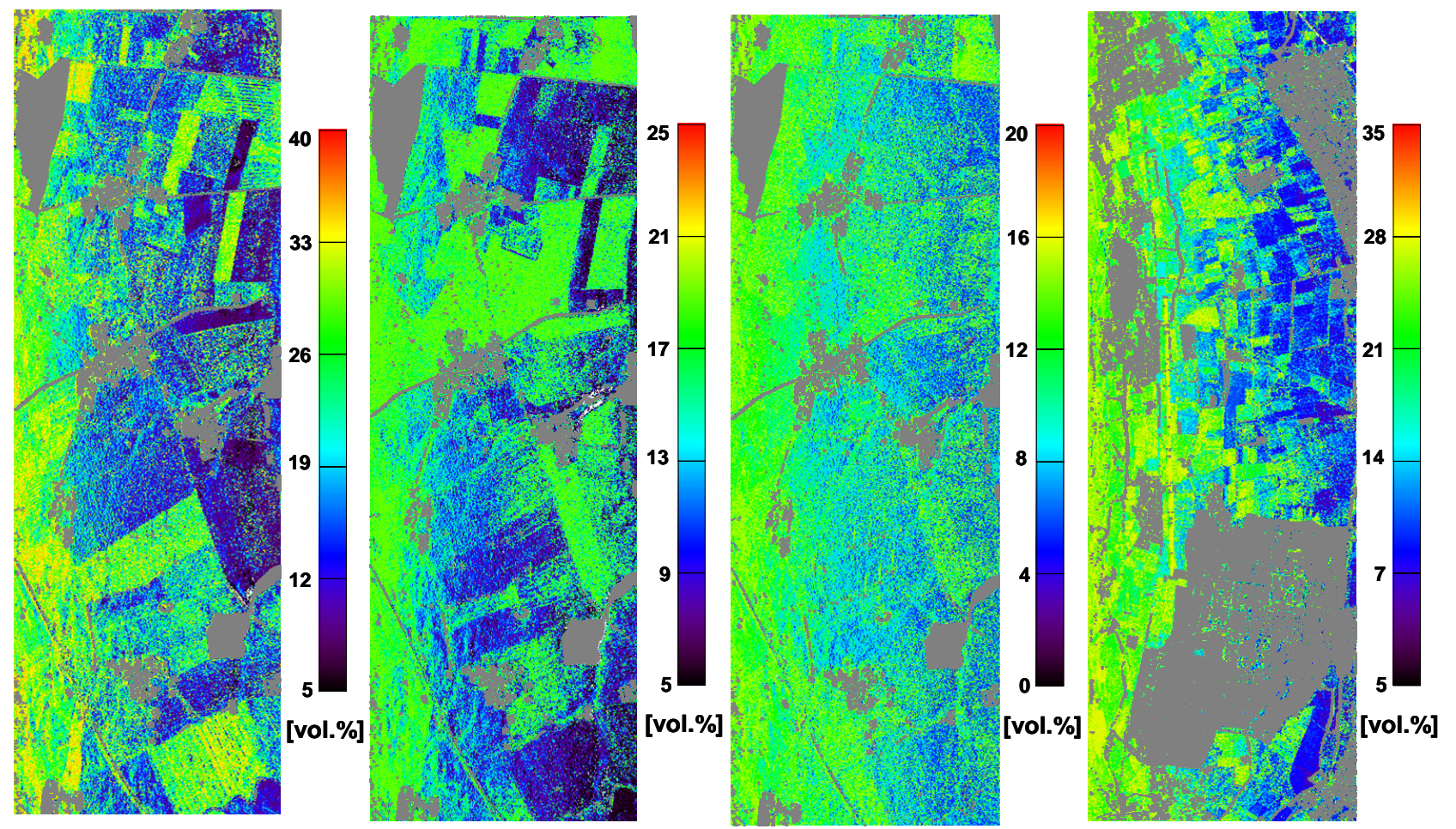

Fig. 3. Results of soil moisture inversion under agricultural vegetation in vol.\% using the hybrid decomposition and inversion approach for the acquisitions (from left to right): AgriSAR in April, June and July 2006, SARTEO in May 2008; White / gray color = Non-invertible / urban and forested areas.
Despite the variety in hydrological and phenological conditions for the four acquisitions, which cover the entire growth season, the soil moisture inversion results in a spatially continuous and complete retrieval under agricultural vegetation cover, where urban and forested areas are masked gray due to model mismatch.

Tab. 1. $\varepsilon_{\text {est }}$-level, inversion rate and maximum of vegetation height for the different dates and acquisitions along the vegetation growth cycle

\begin{tabular}{cccc}
\hline Date & $\begin{array}{c}\varepsilon_{\text {est }} \text {-level } \\
{[-]}\end{array}$ & $\begin{array}{c}\text { Inversion } \\
\text { rate [\%] }\end{array}$ & $\begin{array}{c}\text { Max. vegetation } \\
\text { height [cm] }\end{array}$ \\
\hline $\begin{array}{c}\text { AgriSAR } \\
\text { April 2006 }\end{array}$ & 20 & 99.95 & 18 \\
$\begin{array}{c}\text { AgriSAR } \\
\text { June 2006 } \\
\text { AgriSAR }\end{array}$ & 10 & 99.96 & 167 \\
$\begin{array}{l}\text { July 2006 } \\
\text { SARTEO }\end{array}$ & 8 & 99.94 & 172 \\
May 2008 & 15 & 99.95 & $\sim 70^{1}$ \\
${ }^{1}$ no direct measurements & &
\end{tabular}

The achieved inversion rates are listed in Tab. 1 together with the estimated dielectric level required within the hybrid decomposition and inversion algorithm (see details in [6]) and with the maximum vegetation height, stating a distinct vegetation growth to a mature vegetation cover. 
The quality of the soil moisture inversion under agricultural vegetation cover is assessed by a validation with in situ measurements of soil moisture in Fig. 4. For validation, FDR, TDR and gravimetric probes were used during the field campaigns to quantify the soil moisture content for representative measurement locations on several test fields with different types of winter crops (wheat, rape, barley) and summer crops (sugar beet, corn). A box of 13x13 pixels was considered for each measurement location to realize 169 looks and the mean values of these boxes are compared with the respective in situ measurements to yield the scatter plots for summer (top of Fig. 4) and winter (bottom of Fig. 4) crops.
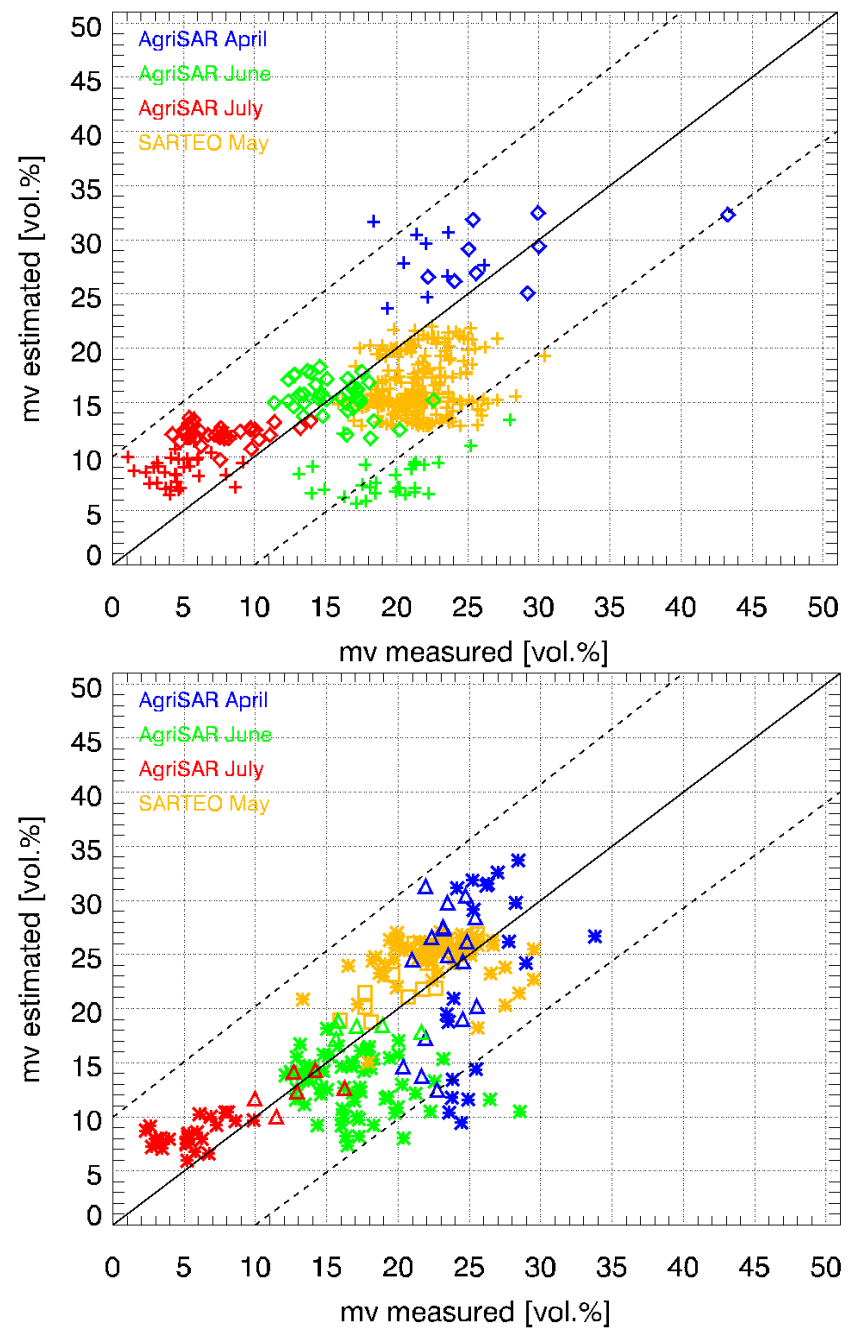

Fig. 4. Validation of estimated soil moisture values for summer crops (top: $+=$ sugar beet, $\diamond=$ corn) and winter crops (bottom: * =wheat, $\Delta=$ rape, $\square=$ barley) with measured soil moisture values from FDR, TDR and gravimetric probes.

The root mean square error for the variety of summer and winter crops amounts to $6.25 \mathrm{vol} . \%$ and $5.77 \mathrm{vol} . \%$ respectively, while a measured soil moisture range from $\sim 2 \mathrm{vol} . \%$ to $\sim 30 \mathrm{vol} . \%$ is covered by the inversion. But the sugar beet fields reveal the most distinct underestimation that might refer to their broad leaf geometry, which is not directly specified in the volume model of the current algorithm.

\section{SUMMARY AND CONCLUSIONS}

Soil moisture is retrieved under agricultural vegetation with a hybrid decomposition and inversion algorithm using fully polarimetric SAR data at L-band. The results for two campaigns reveal a very high inversion rate obtaining a spatially continuous inversion in agricultural areas. The validation with in situ measurements for a variety of summer and winter crops results in a root mean square error of $6.25 \mathrm{vol} . \%$ and $5.77 \mathrm{vol} . \%$. Almost all values in Fig. 4 are located inside the $\pm 10 \mathrm{vol} . \%$-interval (dashed lines), while outliers might require the inclusion of an oriented volume in the hybrid decomposition algorithm.

\section{ACKNOWLEDGMENTS}

The authors would like to thank the AgriSAR, OPAQUE, and SARTEO science teams for their contributions to the campaigns as well as ESA for supporting the activities.

\section{REFERENCES}

[1] W. Wagner, G. Blöschl, P. Pampaloni, J.-C. Calvet, B. Bizzarri, J.-P. Wigneron \& Y. Kerr, "Operational readiness of microwave remote sensing of soil moisture for hydrologic applications," Nordic Hydrology, vol. 38, pp. 1-20, 2007.

[2] I. Hajnsek, T. Jagdhuber, H. Schön \& K.P. Papathanassiou, "Potential of Estimating Soil Moisture under Vegetation Cover by means of PolSAR,” IEEE Trans. Geosci. Remote Sensing, vol. 47, pp. 442-454, 2009.

[3] T. Jagdhuber, H. Schön, I. Hajnsek \& K.P. Papathanassiou, "Soil Moisture Estimation under Vegetation applying Polarimetric Decomposition Techniques," $4^{\text {th }}$ Int. PolInSAR Workshop, Frascati, Italy, January 26-30, p. 1-8, 2009.

[4] S.R. Cloude \& E. Pottier, "A Review of Target Decomposition Theorems in Radar Polarimetry," IEEE Trans. Geosci. Remote Sensing, vol. 34, pp. 498-518, 1996.

[5] S.R. Cloude, "Polarisation: Applications in Remote Sensing," Oxford, Oxford University Press, 2010.

[6] T. Jagdhuber, I. Hajnsek, K.P. Papathanassiou \& A. Bronstert, "A Hybrid Decomposition for Soil Moisture Estimation under Vegetation Cover Using Polarimetric SAR," $5^{\text {th }}$ Int. PolInSAR Workshop, Frascati, Italy, January 24-28, p. 1-6, 2011.

[7] G.C. Topp, J.L. Davis \& A.P. Annan, "Electromagnetic Determination of Soil Water Content: Measurements in Coaxial Transmission Lines,” Water Resources Res., vol. 16, pp. 574-582, 1980.

[8] R. Bianchi, M. Davidson, I. Hajnsek, M. Wooding \& C. Wloczyk, "AgriSAR 2006 - Final report," ESA final report, Noordwijk, The Netherlands, 2008.

[9] I. Hajnsek, T. Jagdhuber \& H. Schön, "SARTEO Data Analysis Report,“ DLR report, Oberpfaffenhofen, Germany, 2009. 